\title{
Jig Design for Window Frame Welding of Rail-Borne Door Systems
}

Ivan Mrkvica, Zbyněk Týn, Tomáš Szotkowski, Jana Petrů, Tomáš Zlámal

Faculty of Mechanical Engineering, VSB-Technical University of Ostrava, Department of Machining, Assembly and Manufacturing Metrology, 17. listopadu 15/2172, 70833 Ostrava-Poruba, Czech Republic. E-mail: ivan.mrkvica@vsb.cz, zbynek.tyn.st@vsb.cz, tomas.szotkowski.st1@vsb.cz, jana.petru@vsb.cz, tomas.zlamal@vsb.cz

Article deals with design of jig for welding of rail-borne door system on the basis of model and drawing of window frame. Using this jig is ensured right setting and reliable clamping of two bent profiles. By welding of these profiles is made a window frame of automatic door systems for rail-bornes. TIG method is used for welding. Article describes and explains setting and clamping of window frame into jig. Design of control jig for window frames of similar type is embodied in text too. Control jig helps by dimension measurement of window frames.

Keywords: welding, jig, basic frame, basic board, clamp

\section{Acknowledgment}

This article has been solved in connection with projects Education system for personal resource of development and research in field of modern trend of surface engineering - surface integrity, reg. no. CZ.1.07/2.3.00/20.0037 financed by Structural Founds of Europe Union and from the means of state budget of the Czech Republic and by project Students Grant Competition SP2016/172 and SP2016/174 financed by the Ministry of Education, Youth and Sports and Faculty of Mechanical Engineering V̌̆B-TUO.

\section{References}

[1] MADDEN, J., STOWELL, M. P., WU, P., LI, H., HE, L. Welding Fixture with Active Position Adapting [Functions. Worchester polytechnic institute, [online] 2007 [cit. 2017-01-18]. Available: https://web.wpi.edu/Pubs/ Eproject/Available/E-project-082307102950/unrestricted/WeldingFixture with_Active_Position_ Adapting_Functions.pdf]

[2] Mechanisation and Welding Fixture: ISF Aachen [online]. 2002 Available: [http://mercury.kau.ac.kr/ welding/Welding\%20Technology\%20I\%2020Welding\%20Processes/Chapter\%2014\%20\%20Mechanisatio\% 20and\%20Welding\%20Fixtures.pdf].

[3] TOKAIENGENEERING PVD.LTD. Welding jigs and fixture [online]. 2016 [cit. 2017-01-18]. Available: [http://tokaiengineering.com/welding-jigs-and-fixtures.html]

[4] INDIAMART. Welding jigs [online]. 2016 [cit. 2017-01-18]. Available: [https://dir.indiamart.com/impact/welding-jigs.html]

[5] MRKVICA, I., SLEHA, V., PETRU, J., NESLUSAN, M., JURKO, J., PANDA, A. (2016) Design of control jig for inserts measurement. In: Manufacturing Technology, Vol. 16, No. 1, p. 198-204.

[6] MRKVICA, I., JANOS, M. (2010) Design of jig for turbo-blower support machining. In: Manufacturing technology, Sp. No., pp. 96-99.

[7] MRKVICA, I., CEP, R., SYSEL, P. (2010) Holding jig proposal for machining of hydraulic hose termination. In: Manufacturing technology, Vol. 15, No. 4, pp. 35-39.

[8] Company IFE-CR. Linkedin [online]. 2016 [cit. 2016-04-04]. Available: [https://www.linkedin.com/company/ifebrno?trk=company_logo]

[9] TYN, Z. (2016) Design of window frame welding jig. Master's thesis, Ostrava: VSB-TU Ostrava, 2016,51 p.

[10] KUBICEK, J. Technology II. - welding: sylabus No 1, [online]. Brno, 2006 [cit. 2016-02-13]. Available: [http://ust.fme.vutbr.cz/svarovani/opory.htm]

[11] Tungsten elektrodes for TIG welding Svarbazar [online]. 2016 [cit. 2016-04-04]. Available: [http://www.svarbazar.cz/phprs/view.php?cisloclanku=2006111201]

[12] [Railway 2016] Railway applications - Welding of rail-bornes and their components. Technical standards ČSN, [online].2016 [cit. 2016-04-04]. Available: [http://www.technicke-normy-csn.cz/284401-csn-en-150853_4_80771.html]

[13] [Standard 2016] Standard used Al alloys. Strojmetal, [online]. 2016 [cit. 2016-04-04]. Available: [http://www.strojmetal.cz/tabulka-slitin] 
[14] Aluminium Corrosion Resistance. Aluminiumdesign [online]. 2016 [cit. 2016-04-04]. Available: [http://www.aluminiumdesign.net/design-support/aluminium-corrosion-resistance/\#toc-galvanic-corrosion]

[15] Different types of corrosion. Corrosionclinic [online]. Webcorr, 1995 [cit. 2016- 04-04]. Available: [http://www.corrosionclinic.com/types_of_corrosion/aluminium_exfoliation_corrosion.htm]

[16] Vertical clamps , Zamet [online]. 2016 [cit. 2016-04-04]. Available: [http://www.zamet.cz/cz/14-detail-produktu/2-svisle/57-svisle-upinky.html]

\section{Paper number: M201795}

Copyright $($ 2017. Published by Manufacturing Technology. All rights reserved. 